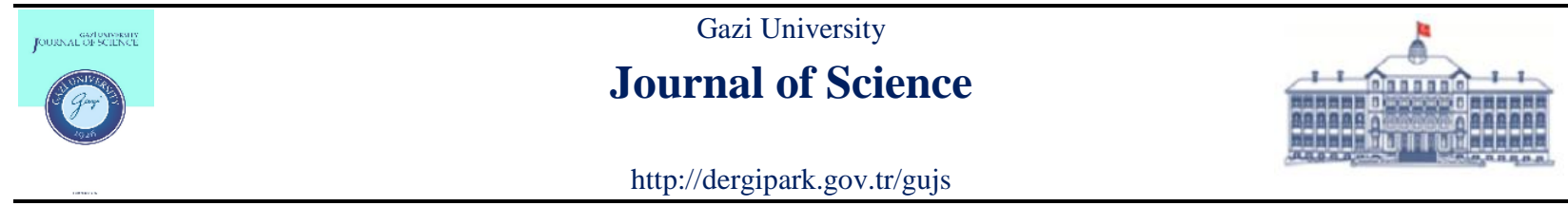

\title{
Hyers-Ulam-Rassias Stability for Abel-Riccati Type First-Order Differential Equations
}

\author{
Yasemin BASCI ${ }^{1, *}$ (i), Suleyman OGREKCI ${ }^{(1)}$, Adil MISIR ${ }^{3}$ \\ ${ }^{1}$ Department of Mathematics, Faculty of Arts and Sciences, Bolu Abant Izzet Baysal University, 14280 Gölköy, Bolu, Turkey. \\ ${ }^{2}$ Department of Mathematics, Faculty of Arts and Sciences, Amasya University, 05000 Amasya, Turkey. \\ ${ }^{3}$ Department of Mathematics, Faculty of Sciences, Gazi University, 06500 Teknikokullar, Ankara, Turkey.
}

\section{Highlights}

- The paper focused on the stability of Hyers-Ulam, Hyers-Ulam-Rassias and Hyers-Ulam-Rassias-Gavruta.

- This is the generalization of many previous studies.

- The equation includes linear, Bernoulli, Riccati and Abel equations.

- It is also the first work related to the stability of Abel equations in the literature.

\section{Article Info}

Received: 07/12/2018

Accepted: 11/04/2019

\section{Keywords}

Abel differential equation

Hyers-Ulam stability

Hyers-Ulam-Rassias stability

Hyers-Ulam-Rassias-Gavruta stability

Riccati differential equation

\begin{abstract}
This paper examines Hyers-Ulam (HU), Hyers-Ulam-Rassias (HUR) and HyersUlam-Rassias-Gavruta (HURG) stability of the first-order differential equation including Bernoulli's, Riccati and Abel with given initial condition.
\end{abstract}

\section{INTRODUCTION}

HU stability is one of the main topics in the theory of functional equations. In 1940, S.M. Ulam posed the problem: "Give conditions in order for a linear mapping near an approximately linear mapping to exist". One year later, Hyers [1] was first answered this problem in the following way:

Let $S=(S,+)$ be an Abelian semigroup and assume that a function $f: S \rightarrow \mathbb{R}$ satisfies the inequality $|f(t+s)-f(t)-f(t)| \leq \varepsilon,(t, s \in S)$ for some positive $\varepsilon$. Then, there exists a unique additive function $g: S \rightarrow \mathbb{R}$ such that $|g(t)-f(t)| \leq \varepsilon,(t \in S)$ holds.

Bourgin [2], Aoki [3] and Rassias [4] were extented the above result of Hyers [1]. Stability problem of differential equations in the sense of HU was initiated by the papers of Obloza [5, 6]. In 1998, Alsina and Ger [7] examined the HU stability for the linear differential equation $y^{\prime}(t)=y(t)$. They proved that if a differentiable function $y: I \rightarrow \mathbb{R}$ satisfies $\left|y^{\prime}(t)-y(t)\right| \leq \varepsilon$, for all $t \in I$, then there exists a differentiable function $f: I \rightarrow \mathbb{R}$ such that $f^{\prime}(t)=f(t)$ and $|y(t)-f(t)| \leq 3 \varepsilon$, for all $t \in I$, where $\varepsilon>0$ and $I$ is an open interval. 
Also, the above result of Alsina and Ger [7] generalized by Miura [8], Miura et al. [9], Takahasi et al. [10] and Miura et al. [11]. Indeed, they presented the HU stability of $y^{\prime}(\mathrm{t})=\lambda y(t)$.

In 2004, Jung [12] gave the HU stability of $\psi(t) y^{\prime}(t)=y(t)$. After, Jung [13], Miura et al. [14] and Takahasi et al. [15] have been generalized the result of the HU stability for first-order linear differential equations. They considered the following nonhomogeneous linear differential equation:

$y^{\prime}(\mathrm{t})+p(t) y(t)+q(t)=0$.

In 2006, Jung [13] investigated the HUR stability of Eq.(1). Also, Jung [16] gave the generalized HU stability of the differential equation of the form

$t y^{\prime}(t)+\alpha y(t)+\beta t^{r} x_{0}=0$.

In 2008, Wang et al. [17] studied the following differential equation:

$r(t) y^{\prime}(\mathrm{t})-p(t) y(t)-q(t)=0$.

Using the method of the integral factor, they proved the HU stability of Eq.(2) and extend the existing results.

In 2008, Jung and Rassias [18] gave the HU stability of the following Riccati equation:

$y^{\prime}(\mathrm{t})+p(t) y(t)+h(t) y^{2}(t)=k(t)$

under the given additional conditions.

In 2009 and 2010, Rus [19,20] presented Ulam-Hyers (UH) stability, generalized UH stability, UlamHyers-Rassias (UHR) stability and generalized UHR stability for the ordinary differential equations

$y^{\prime}(\mathrm{t})=f(t, y(t))$

and

$y^{\prime}(\mathrm{t})=q(t)+f(t, y(t))$.

Also, in 2010, Jung [21] proved that the HUR stability and the HU stability of the Eq.(4) are defined on a finite and closed interval by using the fixed point method and adopting the idea used in Cãdariu and Radu [22].

In 2013, Li and Wang [23] obtained the results of HUR stability and UH stability for the following semilinear differential equation :

$y^{\prime}(t)=\lambda y(t)+f(t, y(t))$.

In 2014, Alqifiary [24] gave a necessary and sufficient condition for the HUR stability and found HU stability constant under certain conditions in order that the linear system of differential equations

$y^{\prime}(t)+A(t) y(t)+B(t)=0$.

In the same year, Qarawani [25] established the stability of first-order linear and nonlinear differential equations in the sense of HUR. Also, he investigated stability and asymptotic stability in the sense of HUR for a differential equation of the form

$y^{\prime}(t)+p(t) y(t)=G(t, y(t))$

and for Bernoulli's differential equation $G(t, \mathrm{y})=\mathrm{q}(\mathrm{t}) y^{n}, \mathrm{n}>1$ with initial condition 
$y\left(t_{0}\right)=y_{0}$

where the continuous function $G(t, y):\left[t_{0}, t_{1}\right] \times \mathbb{R} \rightarrow \mathbb{R}$ satisfies the Lipschitz condition

$|G(t, y)-G(t, z)| \leq L|y-z|$,

for any $t \in\left[t_{0}, t_{1}\right]$, and $y, z \in \mathbb{R}, G(t, 0)=0$, for all $-\infty<t_{0}<t_{1} \leq \infty$.

In 2017, Onitsuka and Shoji [26] gave the HU stability of the following differential equation:

$y^{\prime}(\mathrm{t})-a y(t)=0$,

where $a$ is a nonzero real number. They found an explicit solution $y(t)$ of Eq.(8) satisfying that $|\Phi(t)-y(t)| \leq \frac{\varepsilon}{|a|}$, for all $t \in \mathbb{R}$ under the assumption that a differential function $\Phi(t)$ satisfies $\left|\Phi^{\prime}(t)-a \Phi(t)\right| \leq \varepsilon$, for all $t \in \mathbb{R}$.

Serious studies on the stability problem of differential equations began after 2000's. Stability has been investigated for the different classes of differential equations with different approaches. For example, a special type of ordinary differential equations are delay differential equations. To our knowledge, in 2010, the first mathematicians who investigated the stability of delay differential equations were Jung and J. Brzdek [27]. Enacted by the stated outcomes on HU stability above, they gave the HU stability of $y^{\prime}(t)=$ $\lambda y(t-\tau)$, for $[-\tau, \infty)$ with an initial condition, where $\tau$ and $\lambda$ are positive real constants. Thereafter, Otrocol and Ilea [28] investigated UH stability and generalized UHR for the following delay differential equation:

$y^{\prime}(t)=f(t, y(t), y(g(t)))$.

In 2015, using the fixed point method, Tunç and Biçer [29] obtained new results on the HUR stability and the HU stability for the first-order delay differential equation

$y^{\prime}(t)=F(t, y(t), y(t-\tau))$.

Very recently, the HU stability and the HUR stability have been studied for delay differential equations by $[30,31]$ and the references can be found therein.

The theory of time scale and the related dynamic equations have been systematically studied since last two decades. To our knowledge, in 2013, András and Mészáros [32] first studied the UH stability for some linear and nonlinear dynamic equations on time scales. Additionally, they obtained the UH stability for some integral equations on time scales. They both used direct and operational methods. In 2013, Shen [33] established the Ulam stability for the dynamic equation $y^{\Delta}(t)=q(t) y(t)+f(t)$ and its adjoint equation $x^{\Delta}(t)=-q(t) x^{\sigma}+f(t)$, on a finite interval on the time scale by using the integrating factor method. In the same year, by using the idea of time scale, Zada et al. [34] studied a relationship between the HU stability of the dynamic system $x^{\Delta}(t)=G x(t)$ and its dichotomy. Moreover, for some new studies on the stability of impulsive and fractional differential equations, we recommend the readers to see [35-43].

Our research is inspired by the paper of Qarawani [25]. In this studied, we establish the stability in the sense of HU, HUR and HURG for the following differential equation of the form:

$y^{\prime}(t)+\mathrm{p}(t) y(t)=\sum_{k=0}^{n} p_{k}(t) y^{k}(t)$

with the initial condition

$$
y\left(t_{0}\right)=y_{0},
$$


where $n \geq 0, y(t) \in C^{1}(I), p(t)$ and $p_{k}(t)$ are continuous functions on $I$ for $k=0,1,2, \ldots, n$ and $I=$ $\left[t_{0}, t_{1}\right]$, for $-\infty<t_{0}<t_{1} \leq \infty$. Additionally, $p_{k}(t) y^{k}(t)$ satisfy Lipschitz conditions such that $\left|p_{k}(t) y^{k}(t)-p_{k}(t) z^{k}(t)\right| \leq L_{k}|y-z|$, for any $t \in I$ and $k \in\{2, \ldots, n\}$, where $y, z \in \mathbb{R}$.

Althought this problem can be thought as a special case of [25], when we take $G(t, y)=\sum_{k=0}^{n} p_{k}(t) y^{k}(t)$. But in fact, in [25] $G(t, 0)=0$. However in our case, if the function $p_{0}$ has the property $p_{0}(t) \neq 0$ for some point $t \in\left[t_{0}, t_{1}\right]$, then it requires that $G(t, 0) \neq 0$. For example, if the function $G(t, y)$ is taken as $G(t, y)=$ $1+t y^{2}$, then it becomes $G(t, 0)=1 \neq 0$ and the condition of Qarawani's [25] becomes invalid. Therefore, this refer to the originality of the mentioned problem which is also generalization of some of the previous studies.

\section{DEFINITIONS}

Under the given assumptions in Section 1, we conceive the following nonlinear differential equation:

$y^{\prime}(t)+p(t) y(t)=G(t, y)$

with the initial condition

$y\left(t_{0}\right)=y_{0}$,

where $G(t, y)=\sum_{k=0}^{n} p_{k}(t) y^{k}(t)$. Then, the continuous function $G(t, y):\left[t_{0}, t_{1}\right] \times \mathbb{R} \rightarrow \mathbb{R}$ satisfies the Lipschitz condition $|G(t, y)-G(t, z)| \leq L|y-z|$, for any $t \in\left[t_{0}, t_{1}\right]$ and $y, z \in \mathbb{R},-\infty<t_{0}<t_{1} \leq \infty$.

Definition 1. We say that the Equation (11) has the HU stability if there exists a constant $K>0$ with the following property: For every $\varepsilon>0$, if $y \in C^{1}(I)$ satisfies the inequality

$\left|y^{\prime}(t)+p(t) y(t)-G(t, y)\right| \leq \varepsilon$

with the initial condition (12), then there exists a solution $x(t) \in C^{1}(I)$ of the initial-value problem (11)(12) such that $|y(t)-x(t)| \leq K \varepsilon$.

Definition 2. We say that the Equation (11) has the HUR stability if there exists a constant $K>0$ with the following property: For every $\varepsilon>0$, if $y \in C^{1}(I)$ satisfies the inequality

$\left|y^{\prime}(t)+p(t) y(t)-G(t, y)\right| \leq \varepsilon \varphi(t)$

with the initial condition (12) and $\varphi(t) \in C(I, \mathbb{R})$, then there exists a solution $x(t) \in C^{1}(I)$ of the initialvalue problem (11)-(12) such that $|y(t)-x(t)| \leq K \varepsilon \varphi(t)$, where $K$ is a constant that does not depend on $\varepsilon$ nor $y(t)$.

Definition 3. We say that the Equation (11) has the HURG stability if there exists a constant $K>0$ with the following property: For every $\varepsilon>0, y \in C^{1}(I)$ satisfies the inequality

$\left|y^{\prime}(t)+p(t) y(t)-G(t, y)\right| \leq \varepsilon \varphi(t)$

with the initial condition (12) and $\varphi(t) \in C(I, \mathbb{R})$, then there exists a solution $x(t) \in C^{1}(I)$ of the initialvalue problem (11)-(12) such that $|y(t)-x(t)| \leq K \varepsilon \phi(t)$, where $K$ is a constant that does not depend on $\varepsilon$ nor $y(t)$ and $\phi(t)>0$.

\section{MAIN RESULTS}

Below, we will prove stability and asymptotic stability in the sense of HU, HUR and HURG of Equation (9) with the initial condition (10). 
Theorem 1. If $y(t) \in C^{1}(I), p(t)$ and $p_{k}(t)(k \in\{0,1, \ldots, n\})$ are continuous functions on $I$, then the initial-value problem (9)-(10) has the HU stability.

Proof. Let $y(t)$ be an approximate solution of the Equation (9) with the initial condition (10), and $\varepsilon>0$. We will show that there exists a solution $x(t) \in C^{1}(I)$ of the Equation (9) such that

$|y(t)-x(t)| \leq K \varepsilon$,

where $K>0$ is a constant. Consider the following inequality:

$-\varepsilon \leq y^{\prime}(t)+p(t) y(t)-\sum_{k=0}^{n} p_{k}(t) y^{k}(t) \leq \varepsilon$.

By multiplying of (16) with $e^{\int_{t_{0}}^{t} p(s) d s}$, we obtain

$-\varepsilon e^{\int_{t_{0}}^{t} p(s) d s} \leq\left(y(t) e^{\int_{t_{0}}^{t} p(s) d s}\right)^{\prime}-e^{\int_{t_{0}}^{t} p(s) d s} \sum_{k=0}^{n} p_{k}(t) y^{k}(t) \leq \varepsilon e^{\int_{t_{0}}^{t} p(s) d s}$.

Integrating (17) from $t_{0}$ to $t$, we get

$-\varepsilon \int_{t_{0}}^{t} e^{\int_{t_{0}}^{s} p(\tau) d \tau} d s \leq y(t) e^{\int_{t_{0}}^{t} p(s) d s}-y_{0}-\int_{t_{0}}^{t} e^{\int_{t_{0}}^{s} p(\tau) d \tau} \sum_{k=0}^{n} p_{k}(s) y^{k}(s) d s$

$\leq \varepsilon \int_{t_{0}}^{t} e^{\int_{t_{0}}^{s} p(\tau) d \tau} d s$

Since $p(x)$ is a continuous function on $I=\left[t_{0}, t_{1}\right]$, for all $-\infty<t_{0}<t_{1} \leq \infty$, then it has a maximum on $I$. Let $M=\max \{|p(t)|: t \in I\}$. Hence, we obtain

$-\varepsilon \int_{t_{0}}^{t} e^{M\left(s-t_{0}\right)} d s \leq y(t) e^{\int_{t_{0}}^{t} p(s) d s}-y_{0}-\int_{t_{0}}^{t} e^{\int_{t_{0}}^{s} p(\tau) d \tau} \sum_{k=0}^{n} p_{k}(s) y^{k}(s) d s$

$\leq \varepsilon \int_{t_{0}}^{t} e^{M\left(s-t_{0}\right)} d s$.

From (18), we can write

$-\varepsilon \frac{1}{M}\left(e^{M\left(t-t_{0}\right)}-1\right) \leq y(t) e^{\int_{t_{0}}^{t} p(s) d s}-y_{0}-\int_{t_{0}}^{t} e^{\int_{t_{0}}^{s} p(\tau) d \tau} \sum_{k=0}^{n} p_{k}(s) y^{k}(s) d s$

$\leq \varepsilon \frac{1}{M}\left(e^{M\left(t-t_{0}\right)}-1\right)$.

Now, multiplying (19) with $e^{-\int_{t_{0}}^{t} p(s) d s}$, we get

$-\varepsilon \frac{1}{M}\left(e^{M\left(t-t_{0}\right)}-1\right) e^{-\int_{t_{0}}^{t} p(s) d s} \leq y(t)-e^{-\int_{t_{0}}^{t} p(s) d s}\left(y_{0}+\int_{t_{0}}^{t} e^{\int_{t_{0}}^{s} p(\tau) d \tau} \sum_{k=0}^{n} p_{k}(s) y^{k}(s) d s\right)$

$\leq \varepsilon \frac{1}{M}\left(e^{M\left(t-t_{0}\right)}-1\right) e^{-\int_{t_{0}}^{t} p(s) d s}$,

that is,

$\left|y(t)-e^{-\int_{t_{0}}^{t} p(s) d s}\left(y_{0}+\int_{t_{0}}^{t} e^{\int_{t_{0}}^{s} p(\tau) d \tau} \sum_{k=0}^{n} p_{k}(s) y^{k}(s) d s\right)\right|$ 


$$
\begin{aligned}
& \leq \varepsilon \frac{1}{M}\left(e^{M\left(t-t_{0}\right)}-1\right) e^{-\int_{t_{0}}^{t} p(s) d s} \quad \leq \varepsilon \frac{1}{M} e^{M\left(t-t_{0}\right)} e^{-\int_{t_{0}}^{t} p(s) d s} \\
& \leq \varepsilon \frac{1}{M} e^{M\left(t-t_{0}\right)} e^{\int_{t_{0}}^{t}|p(s)| d s} \quad \leq \varepsilon \frac{1}{M^{2}} e^{2 M\left(t_{1}-t_{0}\right)} .
\end{aligned}
$$

Let us assume that $x(t)$ is given as follows:

$x(t)=e^{-\int_{t_{0}}^{t} p(s) d s}\left(y_{0}+\int_{t_{0}}^{t} e^{\int_{t_{0}}^{s} p(\tau) d \tau} \sum_{k=0}^{n} p_{k}(s) x^{k}(s) d s\right)$.

By using the fundamental theorem of calculus, we get

$$
x^{\prime}(t)=-p(t) e^{-\int_{t_{0}}^{t} p(s) d s}\left(y_{0}+\int_{t_{0}}^{t} e^{\int_{t_{0}}^{s} p(\tau) d \tau} \sum_{k=0}^{n} p_{k}(s) x^{k}(s) d s\right)+\sum_{k=0}^{n} p_{k}(t) x^{k}(t)
$$

and

$x\left(t_{0}\right)=y_{0}$.

Thus, $x(t)$ satisfies the initial-value problem (9)-(10). Now, find the difference:

$$
\begin{aligned}
& |y(t)-x(t)| \leq\left|y(t)-e^{-\int_{t_{0}}^{t} p(s) d s}\left(y_{0}+\int_{t_{0}}^{t} e^{\int_{t_{0}}^{s} p(\tau) d \tau} \sum_{k=0}^{n} p_{k}(s) y^{k}(s) d s\right)\right| \\
& +\mid e^{-\int_{t_{0}}^{t} p(s) d s}\left[\left(y_{0}+\int_{t_{0}}^{t} e^{\int_{t_{0}}^{s} p(\tau) d \tau} \sum_{k=0}^{n} p_{k}(s) y^{k}(s) d s\right)\right. \\
& \left.-\left(y_{0}+\int_{t_{0}}^{t} e^{\int_{t_{0}}^{s} p(\tau) d \tau} \sum_{k=0}^{n} p_{k}(s) x^{k}(s) d s\right)\right] \mid \\
& \leq \varepsilon \frac{1}{M^{2}} e^{2 M\left(t_{1}-t_{0}\right)}+e^{-\int_{t_{0}}^{t} p(s) d s} \int_{t_{0}}^{t} e^{\int_{t_{0}}^{s} p(\tau) d \tau} \sum_{k=1}^{n}\left|p_{k}(s) y^{k}(s)-p_{k}(s) x^{k}(s)\right| d s \\
& \leq \varepsilon \frac{1}{M^{2}} e^{2 M\left(t_{1}-t_{0}\right)}+\int_{t_{0}}^{t} e^{\int_{t}^{s} p(\tau) d \tau} \sum_{k=1}^{n}\left|p_{k}(s)\right|\left|y^{k}(s)-x^{k}(s)\right| d s .
\end{aligned}
$$

Since the derivatives $\left|\frac{\partial\left(p_{k}(t) y^{k}(t)\right)}{\partial y}\right|=\left|k p_{k}(t) y^{k-1}(t)\right|$ are bounded on $S$ for $k \in\{2,3, \ldots, n\}$, the functions $p_{k}(t) y^{k}$ satisfy Lipschitz conditions

$\left|p_{k}(t)\right|\left|y^{k}(t)-x^{k}(t)\right| \leq L_{k}|y(t)-x(t)|$,

where $S=(t, y) \in\left[t_{0}, t_{1}\right] \times\left[-M_{1}, M_{1}\right] \subset \mathbb{R}^{2}$, and since $y \in C^{1}(I)$, there exists a constant $M_{1}$ such that $M_{1}=\max _{t_{0} \leq t \leq t_{1}}|y(t)|$. Moreover, since $p_{1}(t)$ is continuous function on $I$, it is bounded on $I$. Let

$\left|p_{1}(t)\right| \leq L_{1}$.

Therefore, using (20) and (21) we have

$$
|y(t)-x(t)| \leq \varepsilon \frac{1}{M^{2}} e^{2 M\left(t_{1}-t_{0}\right)}+\left(L_{1}+\cdots+L_{n}\right) \int_{t_{0}}^{t} e^{\int_{t}^{s} p(\tau) d \tau}|y(s)-x(s)| d s .
$$

Let

$L=\max \left\{L_{1}, L_{2}, \ldots, L_{n}\right\}$

So, we get 
$|y(t)-x(t)| \leq \varepsilon \frac{1}{M^{2}} e^{2 M\left(t_{1}-t_{0}\right)}+n L \int_{t_{0}}^{t} e^{\int_{t}^{s} p(\tau) d \tau}|y(s)-x(s)| d s$.

By using Gronwall's inequality, we obtain

$$
\begin{aligned}
& |y(t)-x(t)| \leq \varepsilon \frac{1}{M^{2}} e^{2 M\left(t_{1}-t_{0}\right)} e^{n L \int_{t_{0}}^{t} e^{\int_{t}^{S} p(\tau) d \tau} d s} \leq \varepsilon \frac{1}{M^{2}} e^{2 M\left(t_{1}-t_{0}\right)} e^{\frac{n L}{M}\left[1-e^{M\left(t_{0}-t\right)}\right]} \\
& \leq \varepsilon \frac{1}{M^{2}} e^{2 M\left(t_{1}-t_{0}\right)} e^{\frac{n L}{M}}
\end{aligned}
$$

Consequencely,

$\max |y(t)-x(t)| \leq K \varepsilon$,

where $K=\frac{1}{M^{2}} e^{2 M\left(t_{1}-t_{0}\right)} e^{\frac{n L}{M}}$. Therefore, the problem (9)-(10) has the HU stability.

Theorem 2. If $y(t) \in C^{1}(I), p(t)$ and $p_{k}(t)(k \in\{0,1, \ldots, n\})$ are continuous functions on $I$, then the initial-value problem (9)-(10) has the HUR stability.

Proof. Let $\varepsilon>0, \varphi(t)>0$ be an arbitrary function, and $y(t)$ be an approximate solution of the Equation (9) with the initial condition (10). We will show that there exists a solution $x(t) \in C^{1}(I)$ of the Equation (9) such that

$|y(t)-x(t)| \leq K \varepsilon \varphi(t)$

where $K$ is a constant that does not depend on neither $\varepsilon$ nor $y(t)$. Consider the following inequality:

$-\varepsilon \varphi(t) \leq y^{\prime}(t)+p(t) y(t)-\sum_{k=0}^{n} p_{k}(t) y^{k}(t) \leq \varepsilon \varphi(t)$.

Substituting $\varphi(t)=e^{-\int_{t_{0}}^{t} p(s) d s}$ in (23), we have

$-\varepsilon e^{-\int_{t_{0}}^{t} p(s) d s} \leq y^{\prime}(t)+p(t) y(t)-\sum_{k=0}^{n} p_{k}(t) y^{k}(t) \leq \varepsilon e^{-\int_{t_{0}}^{t} p(s) d s}$.

By multiplying (24) with the function $e^{\int_{t_{0}}^{t} p(s) d s}$, we obtain

$-\varepsilon \leq\left(y(t) e^{\int_{t_{0}}^{t} p(s) d s}\right)^{\prime}-e^{\int_{t_{0}}^{t} p(s) d s} \sum_{k=0}^{n} p_{k}(t) y^{k}(t) \leq \varepsilon$.

Integrating (25) from $t_{0}$ to $t$, we get

$-\varepsilon\left(t-t_{0}\right) \leq y(t) e^{\int_{t_{0}}^{t} p(s) d s}-y_{0}-\int_{t_{0}}^{t} e^{\int_{t_{0}}^{s} p(\tau) d \tau} \sum_{k=0}^{n} p_{k}(s) y^{k}(s) d s \leq \varepsilon\left(t-t_{0}\right)$.

Multiplying (26) by the function $e^{-\int_{t_{0}}^{t} p(s) d s}$, we get

$-\varepsilon\left(t-t_{0}\right) e^{-\int_{t_{0}}^{t} p(s) d s} \leq y(t)-e^{-\int_{t_{0}}^{t} p(s) d s}\left(y_{0}+\int_{t_{0}}^{t} e^{\int_{t_{0}}^{s} p(\tau) d \tau} \sum_{k=0}^{n} p_{k}(s) y^{k}(s) d s\right)$

$\leq \varepsilon\left(t-t_{0}\right) e^{-\int_{t_{0}}^{t} p(s) d s}$,

that is,

$\left|y(t)-e^{-\int_{t_{0}}^{t} p(s) d s}\left(y_{0}+\int_{t_{0}}^{t} e^{\int_{t_{0}}^{s} p(\tau) d \tau} \sum_{k=0}^{n} p_{k}(s) y^{k}(s) d s\right)\right| \leq \varepsilon\left(t-t_{0}\right) e^{-\int_{t_{0}}^{t} p(s) d s}$. 
Let us assume that $x(t)$ is given as follows:

$x(t)=e^{-\int_{t_{0}}^{t} p(s) d s}\left(y_{0}+\int_{t_{0}}^{t} e^{\int_{t_{0}}^{s} p(\tau) d \tau} \sum_{k=0}^{n} p_{k}(s) x^{k}(s) d s\right)$.

As it was proved by the above theorem, $x(t)$ satisfies the initial-value problem (9)-(10). Now, find the difference:

$$
\begin{aligned}
& |y(t)-x(t)| \leq\left|y(t)-e^{-\int_{t_{0}}^{t} p(s) d s}\left(y_{0}+\int_{t_{0}}^{t} e^{\int_{t_{0}}^{s} p(\tau) d \tau} \sum_{k=0}^{n} p_{k}(s) y^{k}(s) d s\right)\right| \\
& +\mid e^{-\int_{t_{0}}^{t} p(s) d s}\left[\left(y_{0}+\int_{t_{0}}^{t} e^{\int_{t_{0}}^{s} p(\tau) d \tau} \sum_{k=0}^{n} p_{k}(s) y^{k}(s) d s\right)\right. \\
& \left.-\left(y_{0}+\int_{t_{0}}^{t} e^{\int_{t_{0}}^{s} p(\tau) d \tau} \sum_{k=0}^{n} p_{k}(s) x^{k}(s) d s\right)\right] \mid \\
& \leq \varepsilon\left(t_{1}-t_{0}\right) e^{-\int_{t_{0}}^{t} p(s) d s}+e^{-\int_{t_{0}}^{t} p(s) d s} \int_{t_{0}}^{t} e^{\int_{t_{0}}^{s} p(\tau) d \tau} \sum_{k=1}^{n}\left|p_{k}(s) y^{k}(s)-p_{k}(s) x^{k}(s)\right| d s \\
& \leq \varepsilon\left(t_{1}-t_{0}\right) e^{-\int_{t_{0}}^{t} p(s) d s}+\int_{t_{0}}^{t} e^{\int_{t}^{s} p(\tau) d \tau} \sum_{k=1}^{n}\left|p_{k}(s)\right|\left|y^{k}(s)-x^{k}(s)\right| d s .
\end{aligned}
$$

Employing (20), (21) and (22) in (27), we obtain

$$
|y(t)-x(t)| \leq \varepsilon\left(t_{1}-t_{0}\right) e^{-\int_{t_{0}}^{t} p(s) d s}+n L \int_{t_{0}}^{t} e^{\int_{t}^{s} p(\tau) d \tau}|y(s)-x(s)| d s .
$$

Using Gronwall's inequality, we infer

$$
\begin{aligned}
& |y(t)-x(t)| \leq \varepsilon\left(t_{1}-t_{0}\right) e^{-\int_{t_{0}}^{t} p(s) d s} e^{n L \int_{t_{0}}^{t} e^{\int_{t}^{S} p(\tau) d \tau} d s} \\
& \leq \varepsilon\left(t_{1}-t_{0}\right) e^{-\int_{t_{0}}^{t} p(s) d s} e^{\frac{n L}{M}\left[1-e^{M\left(t_{0}-t\right)}\right]} \quad \leq \varepsilon\left(t_{1}-t_{0}\right) e^{\frac{n L}{M}} e^{-\int_{t_{0}}^{t} p(s) d s} .
\end{aligned}
$$

Hence

$\max |y(t)-x(t)| \leq K \varepsilon \varphi(t)$,

where $\varphi(t)=e^{-\int_{t_{0}}^{t} p(s) d s}$ and $K=\left(t_{1}-t_{0}\right) e^{\frac{n L}{M}}$. Therefore the problem (9)-(10) has the HUR stability.

Theorem 3. If $y(t) \in C^{1}(\mathbb{R}), p(t)$ and $p_{k}(t)(k \in\{0,1, \ldots, n\})$ are continuous functions on $\mathbb{R}$, and $p(t) \geq$ $c>0$, then the initial-value problem (9)-(10) has the HURG stability.

Proof. By using a similar way as the one used in proof of Theorem 2, we got the following inequality:

$$
|y(t)-x(t)| \leq \varepsilon\left(t-t_{0}\right) e^{-\int_{t_{0}}^{t} p(s) d s}+n L \int_{t_{0}}^{t} e^{\int_{t}^{s} p(\tau) d \tau}|y(s)-x(s)| d s .
$$

Using Gronwall's inequality, we obtain

$$
\begin{aligned}
& |y(t)-x(t)| \leq \varepsilon\left(t-t_{0}\right) e^{-\int_{t_{0}}^{t} c d s} e^{n L \int_{t_{0}}^{t} e^{-\int_{s}^{t} c d \tau} d s} \\
& \leq \varepsilon\left(t-t_{0}\right) e^{-c\left(t-t_{0}\right)} e^{n L \int_{t_{0}}^{t} e^{-c(t-s)} d s} \leq \varepsilon\left(t-t_{0}\right) e^{\frac{n L}{c}} e^{-c\left(t-t_{0}\right)} .
\end{aligned}
$$


Hence

$$
\max |y(t)-x(t)| \leq K \varepsilon \varphi(t),
$$

where $K=e^{\frac{n L}{c}}$ and $\phi(t)=\left(t-t_{0}\right) e^{-c\left(t-t_{0}\right)}$. Also, we obtain $\left(t-t_{0}\right) e^{-c\left(t-t_{0}\right)} \rightarrow 0$ as $t \rightarrow \infty$ in (28), whence $\lim _{t \rightarrow \infty}(y(t)-x(t))=0$. Thus, the problem (9)-(10) has the HURG stability.

Remark 1. In Equation (9), substiting some specials values to $n$ and $p_{k}(t)$, as a result it coincides with some preliminary studies in the literature. For example, if we take $n=0,1$ in Equation (9), then Equation (9) are reduced to first-order linear differential equations. There are many studies on these equations in the literature, see $[7,12,13,14,15,16,17,26,28]$. If we take $n=2$ in Equation (9), then Equation (9) are reduced to the Riccati differential equations. So, we achieve the results obtained in [18]. If we take $p_{k}(t)=$ 0 for $k=0,1,2, \ldots, n-1$ in Equation (9), then Equation (9) are reduced to the Bernoulli's differential equations for $n \in \mathbb{N}$. In Equation (9), if $p_{k}(t)=0$ and $n \in(1, \infty)$ are selected for $k=0,1, \ldots, n-1$, performing the similar operations are taken, the results obtained in [25] are reached when $G(t, 0)=0$. If we take $n=3$ in Equation (9), then Equation (9) are reduced to the Abel differential equations. Hence, our work is still valid for Abel differential equations and to our best knowledge there is no such study for this equation in literature. Therefore, this work can be considered as the generalization of many previous works. It is also the first work related to the HU, HUR and HURG stability of Abel equations.

\section{EXAMPLES}

Below, we will give the applications of our main results with three examples.

Example 1. Consider the following initial-value problem:

$$
\begin{aligned}
& y^{\prime}(t)+\frac{2}{t} y(t)=\frac{4}{t^{2}}-4 t^{2} y^{2}(t), \\
& y(1)=1, t \in\left[1, t_{1}\right] .
\end{aligned}
$$

Also, suppose that $y(t)$ is a solution of the following inequality:

$$
\left|y^{\prime}(t)+\frac{2}{t} y(t)+4 t^{2} y^{2}(t)-\frac{4}{t^{2}}\right| \leq \varepsilon
$$

where $(t, y) \in\left[1, t_{1}\right] \times\left[-M_{2}, M_{2}\right], M_{2}=\max _{1 \leq t \leq t_{1}}|y(\mathrm{t})|$. Using the same argument used in Theorem 1 , we can write

$$
\left|y(t)-\frac{1}{t^{2}}\left(1+\int_{1}^{t} s^{2}\left(\frac{4}{s^{2}}-4 s^{2} y^{2}(s)\right) d s\right)\right| \leq \varepsilon\left(\frac{t}{3}-\frac{1}{3 t^{2}}\right) \leq \varepsilon \frac{t_{1}}{3} .
$$

By using the fundamental of calculus, it can be easily shown that

$$
x(t)=\frac{1}{t^{2}}\left(4 t-3-\int_{1}^{t} 4 s^{4} x^{2}(s) d s\right)
$$

satisfies the initial-value problem (29)-(30). Now, find the difference:

$$
\begin{aligned}
& |y(t)-x(t)| \leq\left|y(t)-\frac{1}{t^{2}}\left(4 t-3-\int_{1}^{t} 4 s^{4} y^{2}(s) d s\right)\right| \\
& +\frac{1}{t^{2}} \int_{1}^{t} 4 s^{4}\left|y^{2}(s)-x^{2}(s)\right| d s \\
& \leq \varepsilon \frac{t_{1}}{3}+4 \frac{t_{1}^{4}}{t^{2}} \int_{1}^{t}|y(s)-x(s)||y(s)+x(s)| d s .
\end{aligned}
$$


From $x, y \in\left[-M_{2}, M_{2}\right]$ for $1 \leq t \leq t_{1}$, then we obtain

$|y(s)+x(s)| \leq 2 M_{2}$.

So, for $1 \leq t \leq t_{1}$ we get

$|y(t)-x(t)| \leq \varepsilon \frac{t_{1}}{3}+L \int_{1}^{t}|y(s)-x(s)| d s$,

where $L=8 M_{2} t_{1}^{4}$. Using Gronwall's inequality, we can write

$|y(t)-x(t)| \leq \varepsilon \frac{t_{1}}{3} e^{L \int_{1}^{t} d s}=\varepsilon \frac{t_{1}}{3} e^{L(t-1)} \quad \leq \varepsilon \frac{t_{1}}{3} e^{L t_{1}}=K \varepsilon$,

where $K=\frac{t_{1}}{3} e^{L t_{1}}$. Hence

$\max _{1 \leq t \leq t_{1}}|y(t)-x(t)| \leq K \varepsilon$

So, the initial-value problem (29)-(30) has the HU stability.

Example 2. Consider the initial-value problem:

$y^{\prime}(t)+(\sin t) y(t)=(\sin t) y^{3}(t)+\left(\sin ^{3} t\right) y(t)-\sin t$,

$y(0)=1, t \in\left[0, t_{1}\right]$.

Also, suppose that $y(t)$ is a solution of the following inequality:

$\left|y^{\prime}(t)+(\sin t) y(t)-(\sin t) y^{3}(t)-\left(\sin ^{3} t\right) y(t)+\sin t\right| \leq \varepsilon \varphi(t)$,

where $(t, y) \in\left[0, t_{1}\right] \times\left[-M_{3}, M_{3}\right], M_{3}=\max _{0 \leq t \leq t_{1}}|y(\mathrm{t})|$. Setting $\varphi(t)=e^{\text {cost-1 }}$ in (34), we obtain

$\left|y^{\prime}(t)+(\sin t) y(t)-(\sin t) y^{3}(t)-\left(\sin ^{3} t\right) y(t)+\sin t\right| \leq \varepsilon e^{\cos t-1}$.

Using the same argument used in Theorem 2, we have

$\left|y(t)-e^{\cos t-1}\left(1+\int_{0}^{t} e^{-\cos s+1}\left[(\sin s) y^{3}(s)+\left(\sin ^{3} s\right) y(s)-\sin s\right] d s\right)\right| \leq \varepsilon t_{1} e^{\cos t-1}$.

By using the fundamental of calculus, it can be easily shown that

$x(t)=e^{\cos t-1}\left(1+\int_{0}^{t} e^{-\cos s+1}\left[(\sin s) x^{3}(s)+\left(\sin ^{3} s\right) x(s)-\sin s\right] d s\right)$

satisfies the initial-value problem (32)-(33). Now, find the difference:

$$
\begin{aligned}
& |y(t)-x(t)| \leq\left|y(t)-e^{\cos t-1}\left(1+\int_{0}^{t} e^{-\cos s+1}\left[(\sin s) y^{3}(s)+\left(\sin ^{3} s\right) y(s)-\sin s\right] d s\right)\right| \\
& +e^{\cos t-1} \int_{0}^{t} e^{-\cos s+1}\left|(\sin s) y^{3}(s)+\left(\sin ^{3} s\right) y(s)-(\sin s) x^{3}(s)-\left(\sin ^{3} s\right) x(s)\right| d s \\
& \leq \varepsilon t_{1} e^{\cos t-1}+e^{2} \int_{0}^{t}|\sin s|\left|y^{3}(s)-x^{3}(s)\right| d s+e^{2} \int_{0}^{t}\left|\sin ^{3} s\right||y(s)-x(s)| d s \\
& \leq \varepsilon t_{1} e^{\cos t-1}+e^{2} \int_{0}^{t}|y(s)-x(s)|\left|y^{2}(s)+x^{2}(s)+y(s) x(s)\right| d s+e^{2} \int_{0}^{t}|y(s)-x(s)| d s .
\end{aligned}
$$

From $x, y \in\left[-M_{3}, M_{3}\right]$ for $0 \leq t \leq t_{1}$, then we get 
$\left|y^{2}(s)+x^{2}(s)+y(s) x(s)\right| \leq 3 M_{3}^{2}$.

So, for $0 \leq t \leq t_{1}$ we can write

$|y(t)-x(t)| \leq \varepsilon t_{1} e^{c o s t-1}+L \int_{0}^{t}|y(s)-x(s)| d s$,

where $L=\left(3 M_{3}^{2}+1\right) e^{2}$. Using Gronwall's inequality, we obtain

$|y(t)-x(t)| \leq \varepsilon t_{1} e^{\cos t-1} e^{L \int_{0}^{t} d s}=\varepsilon t_{1} e^{L t} e^{\cos t-1} \leq \varepsilon t_{1} e^{L t_{1}} e^{\cos t-1}=\varepsilon K \varphi(t)$,

where $K=t_{1} e^{L t_{1}}$ and $\varphi(t)=e^{\text {cost-1 }}$. Therefore, we find

$\max _{0 \leq t \leq t_{1}}|y(t)-x(t)| \leq K \varepsilon \varphi(t)$

where $\varphi(t)=e^{\cos t-1}$. So, the problem (32)-(33) has the HUR stability.

Example 3. Consider the Abel differential equation :

$y^{\prime}(t)+2 t y(t)=G(t, y(t))=-\frac{t^{3}}{18} y^{3}(t)+\frac{t^{2}}{6} y^{2}(t)$,

with the initial condition

$y(1)=6, t \in\left[1, t_{1}\right]$.

So, from $p(t)=2 \mathrm{t} \geq 2 \geq c>0$ we choose $c=2$. Because of the derivative $\left|\frac{\partial \mathrm{G}(\mathrm{t}, \mathrm{y})}{\partial \mathrm{y}}\right|$ is bounded on $U$, then $G(t, y(t))=-\frac{t^{3}}{18} y^{3}+\frac{t^{2}}{6} y^{2}$ satisfies the Lipschitz condition $|G(t, y)-G(t, z)| \leq L|y-z|$, for all $(t, y),(t, z) \in U$, where $U=(t, y) \in\left[1, t_{1}\right] \times\left[-M_{4}, M_{4}\right], M_{4}=\max _{1 \leq t \leq t_{1}}|y(\mathrm{t})|$. Also, suppose that $y(t)$ is a solution of the following inequality:

$-\varepsilon \varphi(t) \leq y^{\prime}(t)+2 \operatorname{ty}(t)+\frac{t^{3}}{18} y^{3}(t)-\frac{t^{2}}{6} y^{2}(t) \leq \varepsilon \varphi(t)$.

Substituting $\varphi(t)=e^{1-t^{2}}$ in (37), we get

$-\varepsilon e^{1-t^{2}} \leq y^{\prime}(t)+2 \mathrm{t} y(t)+\frac{t^{3}}{18} y^{3}(t)-\frac{t^{2}}{6} y^{2}(t) \leq \varepsilon e^{1-t^{2}}$.

Multiplying (38) by $e^{t^{2}-1}$, we obtain

$-\varepsilon \leq\left(y(t) e^{t^{2}-1}\right)^{\prime}+e^{t^{2}-1}\left(\frac{t^{3}}{18} y^{3}(t)-\frac{t^{2}}{6} y^{2}(t)\right) \leq \varepsilon$.

Integrating (39) from 1 to $t$ and multiplying by $e^{1-t^{2}}$, we have

$-\varepsilon(t-1) e^{1-t^{2}} \leq y(t)-e^{1-t^{2}}\left(6+\int_{1}^{t} e^{s^{2}-1}\left(\frac{s^{2}}{6} y^{2}(s)-\frac{s^{3}}{18} y^{3}(s)\right) d s\right)$

$\leq \varepsilon(t-1) e^{1-t^{2}}$.

By using the fundamental of calculus, it can be easily shown that

$x(t)=e^{1-t^{2}}\left(6+\int_{1}^{t} e^{s^{2}-1}\left(\frac{s^{2}}{6} x^{2}(s)-\frac{s^{3}}{18} x^{3}(s)\right) d s\right)$ 
satisfies the initial-value problem (35)-(36). Now, find the difference:

$$
\begin{aligned}
& |y(t)-x(t)| \leq\left|y(t)-e^{1-t^{2}}\left(6+\int_{1}^{t} e^{s^{2}-1}\left(\frac{s^{2}}{6} y^{2}(s)-\frac{s^{3}}{18} y^{3}(s)\right) d s\right)\right| \\
& +e^{-t^{2}+1} \int_{1}^{t} e^{s^{2}-1}\left|\frac{s^{2}}{6} y^{2}(s)-\frac{s^{3}}{18} y^{3}(s)-\frac{s^{2}}{6} x^{2}(s)+\frac{s^{3}}{18} x^{3}(s)\right| d s \\
& \leq\left|y(t)-e^{-t^{2}+1}\left(6+\int_{1}^{t} e^{s^{2}-1}\left(\frac{s^{2}}{6} y^{2}(s)-\frac{s^{3}}{18} y^{3}(s)\right) d s\right)\right| \\
& +e^{-t^{2}+1} \int_{1}^{t} e^{t^{2}-1}\left|\frac{s^{2}}{6} y^{2}(s)-\frac{s^{3}}{18} y^{3}(s)-\frac{s^{2}}{6} x^{2}(s)+\frac{s^{3}}{18} x^{3}(s)\right| d s \\
& \leq\left|y(t)-e^{-t^{2}+1}\left(6+\int_{1}^{t} e^{t^{2}-1}\left(\frac{s^{2}}{6} y^{2}(s)-\frac{s^{3}}{18} y^{3}(s)\right) d s\right)\right| \\
& +e^{-t^{2}+1} e^{t^{2}-1} \int_{1}^{t}\left|\frac{s^{2}}{6} y^{2}(s)-\frac{s^{3}}{18} y^{3}(s)-\frac{s^{2}}{6} x^{2}(s)+\frac{s^{3}}{18} x^{3}(s)\right| d s \\
& \leq \varepsilon(t-1) e^{1-t^{2}}+\int_{1}^{t}\left|\frac{s^{2}}{6}\right|\left|y^{2}(s)-x^{2}(s)\right| d s+\int_{1}^{t}\left|\frac{s^{3}}{18}\right|\left|y^{3}(s)-x^{3}(s)\right| d s \\
& \leq \varepsilon(t-1) e^{1-t^{2}}+\frac{t_{1}^{2}}{6} \int_{1}^{t}|y(s)-x(s)||y(s)+x(s)| d s \\
& +\frac{t_{1}^{3}}{18} \int_{1}^{t}|y(s)-x(s)|\left|y^{2}(s)+x^{2}(s)+y(s) x(s)\right| d s .
\end{aligned}
$$

From $x, y \in\left[-M_{4}, M_{4}\right]$ for $1 \leq t \leq t_{1}$, then we have

$|y(s)+x(s)| \leq 2 M_{4}$

and

$\left|y^{2}(s)+x^{2}(s)+y(s) x(s)\right| \leq 3 M_{4}^{2}$.

So, for $1 \leq t \leq t_{1}$ we get

$$
\begin{aligned}
& |y(t)-x(t)| \leq \varepsilon(t-1) e^{1-t^{2}}+\left(2 M_{4} \frac{t_{1}^{2}}{6}+3 M_{4}^{2} \frac{t_{1}^{3}}{18}\right) \int_{1}^{t}|y(s)-x(s)| d s \\
& \leq \varepsilon(t-1) e^{1-t^{2}}+M_{5} \int_{1}^{t}|y(s)-x(s)| d s,
\end{aligned}
$$

where $M_{5}=M_{4} \frac{t_{1}^{2}}{3}+M_{4}^{2} \frac{t_{1}^{3}}{6}$. Using Gronwall's inequality, we obtain

$|y(t)-x(t)| \leq \varepsilon(t-1) e^{1-t^{2}} e^{M_{5} \int_{1}^{t} d s} \leq \varepsilon t e^{1-t^{2}} e^{M_{5} t}=\varepsilon K \phi(t)$,

where $K=1$ and $\phi(t)=t e^{1-t^{2}+M_{5} t}$.

Now sending $t \rightarrow \infty$ in (40), we find $\lim _{t \rightarrow \infty} \phi(t)=\lim _{t \rightarrow \infty} t e^{1-t^{2}+M_{5} t}=0$, whence

$\lim _{t \rightarrow \infty}(y(t)-x(t))=0$ 
Thus, the problem (35)-(36) has the HURG stability.

\section{CONCLUSION}

In this study, we have obtained some new results based on the stability in the sense of HU, HUR and HURG for the first-order linear and nonlinear differential equations including Bernoulli's, Riccati and Abel differential equations under the given initial condition. We have removed the strong condition $G(t, 0)=0$ unlike in [25]. Thus, the considered problem appears as an original problem. But in case of $G(t, y)=$ $\mathrm{q}(\mathrm{t}) y^{n}, 0<n<1$ and $n<0$, there exists still an open problem. Finally, we carefully analyzed three illustrative examples.

\section{CONFLICTS OF INTEREST}

No conflict of interest was declared by the authors.

\section{REFERENCES}

[1] Hyers, D.H., "On the stability of the linear functional equation", Proceeding of the National Academy of Sciences of the United States of America, 27: 222-224, (1941).

[2] Bourgin, D.G., "Classes of transformations and bordering transformations", Bulletin of the American Mathematical Society, 57: 223-237, (1951).

[3] Aoki, T., "On the stability of the linear transformation in Banach spaces", Journal of Mathematical Society of the Japan, 2: 64-66, (1950).

[4] Rassias, Th.M. "On the stability of the linear mapping in Banach spaces", Proceeding American Mathematical Society, 72(2): 297-300, (1978).

[5] Obloza, M., "Hyers stability of the linear differential equation", Rocznik Nauk-Dydakt Prace Matematik, 13(1): 259-270, (1993).

[6] Obloza, M., "Connections between Hyers and Lyapunov stability of the ordinary differential equations", Rocznik Nauk-Dydakt Prace Matematik, 14(1): 141-146, (1997).

[7] Alsina, C., Ger, R., "On some inequalities and stability results related to the exponential function", Journal of Mathematical Inequalities, 2(4): 373-380, (1998).

[8] Miura, T., "On the Hyers-Ulam stability of a differentiable map", Scientiae Mathematicae Japonicae, 55: 17-24, (2002).

[9] Miura, T., Takahasi, S.E., Choda, H., "On the Hyers-Ulam stability of real continuous function valued differentiable map", Tokyo Journal of Mathematics, 24: 467-476, (2001).

[10] Takahasi, S.E., Miura, T., Miyajima, S., "On the Hyers-Ulam stability of the Banach space-valued differential equation $y^{\prime}=\lambda y$ ”, Bulletin of Korean Mathematical Society, 39: 309-315, (2002).

[11] Miura, T., Jung, S.M., Takahasi, S.E., "Hyers-Ulam-Rassias stability of the Banach space valued linear differential equations $y^{\prime}=\lambda y$ ", Journal of the Korean Mathematical Society, 41: 995-1005, (2004). 
[12] Jung, S.M., "Hyers-Ulam stability of linear differential equations of first order", Applied Mathematics Letters, 17(10): 1135-1140, (2004).

[13] Jung, S.M., "Hyers-Ulam stability of linear differential equations of first order II", Applied Mathematics Letters, 19(9): 854-858, (2006).

[14] Miura, T., Miyajima, S., Takahasi, S.E., "A characterization of Hyers-Ulam stability of first order linear differential operators", Journal of Mathematical Analysis Applications, 286: 136-146, (2003).

[15] Takahasi, S.E., Takagi, H., Miura, T., Miyajima, S., “The Hyers-Ulam stability constants of first order linear differential operators", Journal of Mathematical Analysis Applications, 296: 403-409, (2004).

[16] Jung, S.M., "Hyers-Ulam stability of linear differential equations of first order III", Journal of Mathematical Analysis Applications, 311(1): 139-146, (2005).

[17] Wang, G., Zhou, M., Sun, L., "Hyers-Ulam stability of linear differential equations of first order", Applied Mathematics Letters, 21: 1024-1028, (2008).

[18] Jung, S.M., Rassias, T.M., "Generalized Hyers-Ulam stability of Riccati differential equation", Mathematical Inequalities and Applications, 11(4): 777-782, (2008).

[19] Rus, I.A., "Ulam stability of ordinary differential equations", Studia Universitatis "Babeş-Bolyai”, Mathematica., 54(4): 125-134, (2009).

[20] Rus, I.A., "Ulam stabilities of ordinary differential equations in a Banach space", Carpathian Journal of Mathematics, 26(1): 103-107, (2010).

[21] Jung, S.M., "A fixed point approach to the stability of differential equations $y^{\prime}=F(x, y)$ ", Bulletin of the Malaysian Mathematical Sciences Society, (2) 33(1): 47-56, (2010).

[22] Cãdariu, L., Radu, V., “On the Stability of the Cauchy Functional Equation: A Fixed Point Approach”, Grazer Mathematische Berichte, 346: 43-52, (2004).

[23] Li, X., Wang, J., "Ulam-Hyers-Rassias stability of semilinear differential equations with impulses", Electronic Journal of Differential Equations, 2013(172): 1-8, (2013).

[24] Alqifiary, Q.H., "Note on the stability for linear systems of differential equations", International Journal of Applied Mathematical Research, 3(1): 15-22, (2014).

[25] Qarawani, M.N.,“On Hyers-Ulam-Rassias stability for Bernoulli's and first order linear and nonlinear differential equations", British Journal of Mathematics and Computer Science, 4(11): 1615-1628, (2014).

[26] Onitsuka, M., Shoji, T.,"Hyers-Ulam stability of first-order homogeneous linear differential equations with a real-valued coefficient", Applied Mathematics Letters, 63: 102-108, (2017).

[27] Jung, S.M., Brzdek, J., "Hyers-Ulam stability of the delay equation $y^{\prime}(t)=\lambda y(t-\tau)$ ",Abstract and Applied Analysis 2010, Article ID 372176, 10 pages (2010).

[28] Otrocol, D., Ilea, V., "Ulam stability for a delay differential equation", Central European Journal of Mathematics, 7: 1296-1303, (2013). 
[29] Tunç, C., Biçer, E., "Hyers-Ulam-Rassias stability for a first-order functional differential equation", Journal of Mathematical and Fundamental Sciences, 47(2): 143-153, (2015).

[30] Zada, A., Shah, S.O., "Hyers-Ulam stability of first-order non-linear delay differential equations with fractional integrable impulses", Hacettepe Journal of Mathematics and Statistics, 47(5): 1196-1205, (2018).

[31] Zada, A., Ali, W., Park, C., "Ulam's type stability of higher order nonlinear delay differential equations via integral inequality of Grönwall- Bellman-Bihari’s type", Applied Mathematics and Computation, 350: 60-65, (2019).

[32] András, S., Mészáros, A.R., "Ulam-Hyers stability of dynamic equations on time scales via Picard operators", Applied Mathematics and Computation, 219: 4853-4864, (2013).

[33] Shen, Y., "The Ulam stability of first order linear dynamic equations on time scales", Results in Mathematics, Online first. (2017), 2017 Springer International Publishing AG DOI 10.1007/s00025017-0725-1.

[34] Zada, A., Shah, S.O., Ismail, S., Li, T., "Hyers-Ulam stability in terms of dichotomy of first order linear dynamic systems", Punjab University Journal of Mathematics, 49(3): 37-47, (2017).

[35] Ali, Z., Zada, A., Shah, K., "Existence and stability analysis of three point boundary value problem", International Journal of Applied Computational Mathematics, 3(1): 651- 664, (2017).

[36] Ali, A., Rabiei, F., Shah,K., "On Ulam's type stability for a class of impulsive fractional differential equations with nonlinear integral boundary conditions", Journal of Nonlinear Sciences and Applications, 10: 4760-4775, (2017).

[37] Shah, K., Tunç, C., "Existence theory and stability analysis to a system of boundary value problem", Journal of Taibah University for Science, 11: 1330-1342, (2017).

[38] Wang, J., Shah, K., Ali, A., "Existence and Hyers-Ulam stability of fractional Nonlinear Impulsive witched coupled evolution equations", Mathematical Methods in the Applied Sciences, 41(6): 23922402, (2018).

[39] Wang, J., Zada, A., Ali, W., "Ulam's-type stability of first-order impulsive differential equations with variable delay in quasi-Banach spaces", International Journal of Nonlinear Sciences and Numerical Simulation, 19(5): 553-560, (2018).

[40] Wang, X., Arif, M., Zada, A., " $\beta$-Hyers-Ulam-Rassias stability of semilinear nonautonomous impulsive system", Symmetry, 11(231): 18 pages, (2019).

[41] Zada, A., Ali, S., "Stability analysis of multi-point boundary value problem for sequential fractional differential equations with non-instantaneous impulses", International Journal of Non-linear Sciences and Numerical Simulation, 19(7): 763-774, (2018).

[42] Zada, A., Yar, M., Li, T., "Existence and stability analysis of nonlinear sequential coupled system of Caputo fractional differential equations with integral boundary conditions", Annales Universitatis Paedagogicae Cracoviensis Studia Mathematica, 17: 103-125, (2018).

[43] Zada, A., Shaleena, S., Li, T., "Stability analysis of higher order nonlinear differential equations in $\beta$ normed spaces", Mathematical Methods in the Applied Sciences, 42(4): 1151-1166, (2019). 